\title{
Ion acceleration with mixed solid targets interacting with circularly polarized lasers
}

\author{
Xiaomei Zhang, ${ }^{1}$ Baifei Shen,,${ }^{1}$ Liangliang Ji, ${ }^{1}$ Fengchao Wang, ${ }^{1}$ Zhangying Jin, ${ }^{1}$ Xuemei Li, ${ }^{1}$ \\ Meng Wen, ${ }^{1}$ and John R. Cary ${ }^{2,3}$ \\ ${ }^{1}$ State Key Laboratory of High Field Laser Physics, Shanghai Institute of Optics and Fine Mechanics, Chinese Academy of Sciences, \\ P.O. Box 800-211, Shanghai 201800, China \\ ${ }^{2}$ Department of Physics, University of Colorado, Boulder, Colorado 80309, USA \\ ${ }^{3}$ Tech-X Corporation, Boulder, Colorado 80303, USA \\ (Received 3 November 2008; published 23 February 2009)
}

\begin{abstract}
The interaction of a circularly polarized laser pulse with a mixed solid target containing two species of ions is studied by particle in cell simulations and analytical model. After the interaction tends to be stable, it is demonstrated that the acceleration is more efficient for the heavier ions than that in plasmas containing a single kind of heavy ion and the acceleration efficiency is higher when its proportion is lower. To obtain monoenergetic heavy-ion beams, a sandwich target with a thin mixed ion layer between two light ion layers and a microstructured target are proposed. The influences of parameters of the laser pulse and target on ion acceleration are discussed in detail. It is found that, when the target is thick enough, a cold target is more appropriate for heavy-ion acceleration than a warm target, and the velocity of the reflected heavy ions is proportional to the laser amplitude.
\end{abstract}

DOI: 10.1103/PhysRevSTAB.12.021301

PACS numbers: 52.38.Kd, 41.75.Jv, 52.65.Rr, 52.27.Cm

\section{INTRODUCTION}

Ion acceleration in laser-driven plasmas has become a hot research topic for its wide applications in many fields, such as cancer therapy and fast ignition of inertial confinement fusion [1]. In the previous studies, various schemes have been proposed for accelerating protons or heavier ions to high energies by using the recently available high-intensity short-pulse lasers. A linearly polarized (LP) laser pulse is often used because it can heat the plasmas quickly and the generated hot electrons are necessary for two well-known acceleration mechanisms: the front shock acceleration [2-6] and target normal sheath acceleration (TNSA) [7-11]. With the latter method, protons up to $58 \mathrm{MeV}$ have been obtained with $3 \times$ $10^{20} \mathrm{~W} \mathrm{~cm}^{-2}$ lasers [12,13]. At the rear surface of the layered thin foils quasimonoenergetic protons and heavy ions are produced in both experiment [8,9,14-17] and theory $[18,19]$. Quasimonoenergetic proton or ion bunches with an energy spread of about $20 \%$ have been produced $[8,9]$. Other promising schemes, such as direct laserpressure acceleration $[20,21]$ and plasma wake acceleration [22] are also extensively explored. Recently, ion acceleration by a circularly polarized (CP) laser pulse has attracted much attention because there is no apparent selfheating mechanism for plasmas and ions can be accelerated stably by the electrostatic field due to the charge separation in the front of the target [23-27]. This mecha-

\footnotetext{
*Author to whom correspondence should be addressed. bfshen@mail.shcnc.ac.cn
}

nism is the so-called electrostatic shock acceleration. In the case of multispecies ion plasmas, protons are especially accelerated more efficiently due to their highest chargemass ratio. For example, in the heavy-ion-dominated plasmas [28], protons are accelerated with a higher efficiency by high-intensity short-pulse CP laser when the heavy-ion mass is much larger. In the proton-dominated plasmas [6], with certain parameters, protons can be accelerated up to twice the shock wave velocity by the LP laser pulse induced electrostatic shock wave, while the heavy ions cannot be accelerated as efficiently as protons. However, in the heavy-ion-dominated plasmas, both protons and heavy ions can be accelerated up to twice the shock wave velocity.

Recently, we have reported a method for efficient generation of heavy-ion bunches from a target containing two species of ions irradiated by a CP laser pulse and monoenergetic heavy ions observation from a sandwich target [29]. Here, this work is devoted to an extended investigation of the acceleration of mixed target. In general, heavy ions cannot be accelerated as efficiently as protons since the electric force per unit mass on them (with lower chargemass ratio) is smaller. Here, the cold two-species-ion plasma is irradiated by a normal intense CP laser pulse. It is found that heavy ions are accelerated more efficiently in the proton-dominated plasmas compared with those in the pure-heavy-ion plasmas. The velocity of heavy ions increases with the rise of the proton proportion. In Sec. II, the detailed acceleration process of the cold mixed targets and the influences of parameters of the laser pulse and target are investigated in detail. The influences of the 
background temperature, target thickness, and laser intensity are discussed in Sec. III. Section IV contains the summary.

\section{ACCELERATION OF COLD MIXED TARGETS}

\section{A. Acceleration process with fixed charge-mass ratio}

For a target containing two species of ions irradiated by a laser pulse, the conservations of momentum and energy yield $[25,30]$

$$
\begin{aligned}
I / c= & -\eta I / c+n_{e 1} A_{1} / Z_{1} m_{p} v_{i 1} v_{s 1} \\
& +n_{e 2} A_{2} / Z_{2} m_{p} v_{i 2} v_{s 2}, \\
I= & \eta I+n_{e 1} A_{1} / Z_{1} m_{p} v_{i 1}^{2} v_{s 1} / 2 \\
& +n_{e 2} A_{2} / Z_{2} m_{p} v_{i 2}{ }^{2} v_{s 2} / 2,
\end{aligned}
$$

where $I$ and $\eta$ are the intensity and reflectivity of the laser pulse, $c$ is the velocity of light in vacuum, $m_{p}$ is the proton mass, $v_{i 1, i 2}$ and $v_{s 1, s 2}$ are the velocities of the reflected ions and the laser induced electrostatic shocks, $Z_{1,2}$ and $A_{1,2}$ are the ion charge and mass number, $n_{e 1} \equiv Z_{1} n_{i 1}, n_{i 1}$ and $n_{e 2} \equiv Z_{2} n_{i 2}, n_{i 2}$ are the corresponding electron and ion densities of each species, respectively. The subscripts 1 and 2 denote the light and heavy-ion species. To see clearly the influence of larger mass, we first set $A_{1}=Z_{1}=1$ for simplification, then $n_{e 1} \equiv n_{i 1}, m_{1}=m_{p} ; Z_{2}=1, A_{2}=$ 10 , then $n_{e 2} \equiv n_{i 2}, m_{2}=A_{2} m_{p}$. It is convenient to use dimensionless variables $\quad v_{i 1, i 2} / c \rightarrow v_{i 1, i 2}, \quad v_{s 1, s 2} / c \rightarrow$ $v_{s 1, s 2}, n_{i 1, i 2} / n_{c} \rightarrow n_{i 1, i 2}, m_{1,2} / m_{e} \rightarrow m_{1,2}$, where $n_{c}$ is the critical density and $m_{e}$ is the electron mass. Assuming that all ions are reflected by the electrostatic shock, after normalization, Eq. (1) is rewritten as

$$
\begin{aligned}
& (1+\eta) a^{2}=2 n_{i 1} m_{1} v_{s 1}^{2}+2 n_{i 2} m_{2} v_{s 2}^{2} \\
& (1-\eta) a^{2}=2 n_{i 1} m_{1} v_{s 1}^{3}+2 n_{i 2} m_{2} v_{s 2}^{3}
\end{aligned}
$$

where the relation $v_{i 1, i 2}=2 v_{s 1, s 2}$ is used. The normalized laser amplitude $a=e A_{0} / m_{e} c^{2}$, where $A_{0}$ is the vector potential, and $e$ is the electron charge. Since the accelera- tion field moves as a constant velocity during the interaction, $v_{s 1}=v_{s 2}=v_{s}$ is set, so that one obtains

$$
v_{s}^{2}+v_{s}^{3}=\frac{a^{2}}{n_{i 1} m_{1}+n_{i 2} m_{2}},
$$

where $n_{i 1}+n_{i 2}=n_{e}$.

As an example, in the one-dimensional particle in cell (1D PIC) simulation code VORPAL [31], we consider the situation that a cold mixed target of initial density $n_{e}=10$ is irradiated normally by a CP laser pulse of wavelength $\lambda=1 \mu \mathrm{m}$ from the left boundary of the simulation box. The target occupies a region between $x=64 \lambda$ and $x=$ $72 \lambda$ initially. The laser amplitude rises from zero to $a=2$ in $5 T$ and then remains constant, where $T$ is the laser period. The proton mass is $m_{i 1}=1836$ and the heavy-ion mass is $m_{i 2}=10 m_{i 1}=18360$.

In the case of single species plasma, the reflected ion velocities are $v_{i 1}=0.0295$ [25] for $m_{i 1}=1836$ and $v_{i 1}=$ 0.0094 for $m_{i 1}=18360$, respectively, from the simulations (see Fig. 4), where the velocity is normalized by $c$. They both agree well with the analytical results $v_{i}=2 u_{s}$ according to the simple model $[5,25,32]$, which is often used to evaluate the shock velocity, or the recessing surface velocity of the single species plasmas as $u_{s} \sim \sqrt{a^{2} /\left(m_{i} n_{i}\right)}$, where $n_{i}$ is the ion density. The simulation results in the case of the proton-dominated plasmas with $n_{i 1}=8, n_{i 2}=$ 2 , i.e., the density ratio $n_{i 1} / n_{i 2}=4$ at two instants are shown in Fig. 1. Here, two different acceleration stages can be seen. At $t=80 T$, protons are accelerated efficiently up to 0.0295 by the electrostatic field while the heavy ions almost remain immobile due to their larger inertia. Protons move ahead and separate from the heavy ions. Subsequently, at $t=180 T$, the heavy ions overtake protons and the reflected protons and heavy ions tend to move at the same velocity approximately, i.e., the velocity of heavy ions increases to 0.018 from 0.0094 and the velocity of protons decreases to 0.018 from 0.0295 . In this stage, the reflected heavy ions have been accelerated by a much more partial electrostatic field so that they are accelerated efficiently. On the contrary, the reflected protons are ahead of
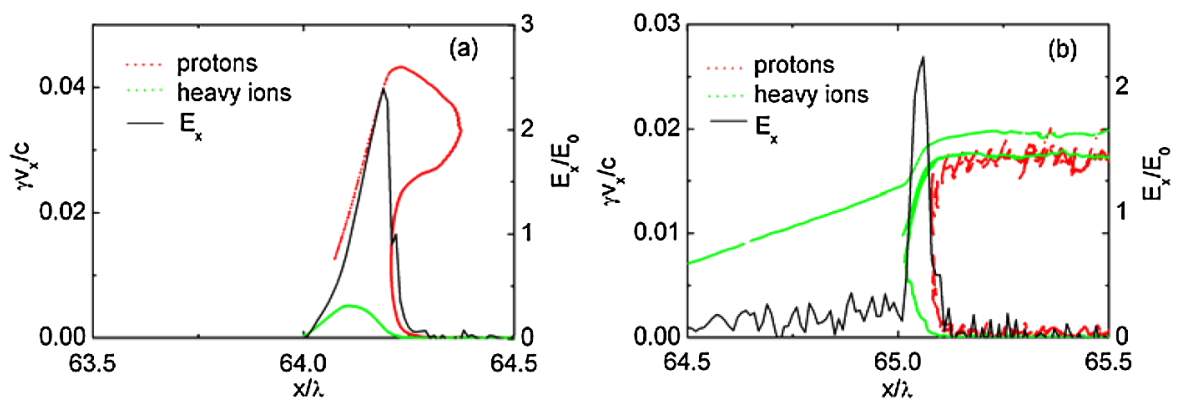

FIG. 1. (Color) Phase space of protons (red dots) and heavy ions (green dots) and the electric field distribution (black solid line) for the proton-dominated plasmas in the case of the density ratio $n_{i 1} / n_{i 2}=4$ at (a) $t=80 T$ and (b) $t=180 T$, respectively. 
heavy ions due to their smaller mass so that they have been accelerated only by a much less partial electrostatic field, resulting in the decrease of the velocity. Therefore, according to the above simulation results, the acceleration process for a mixed solid target containing two species of ions with different mass can also be concluded as follows: first, protons with larger charge-mass ratio tend to move faster and the heavy ions slower, and then the faster (slower) ions will be pulled back (pushed forward) by the space-chargefield redistribution. Finally, the entire system moves together at the same velocity, so heavy ions are drawn by light ions indirectly. This process explains why the light ions are slightly ahead of the heavy ions and the electrostatic field distribution presents a tiny valley between the two reflected ion fronts in Fig. 1(a) of Ref. [29]. Compared with the case of Fig. 1 in this paper, this valley is more obvious due to the relatively more intense laser. The light and heavy ions have the same final velocities because they and the accelerating electric field belong to the same selfconsistent electrostatic shock system, whose velocity is self-consistently determined by the laser and plasma parameters. In this simulation, the acceleration field is dominated by a single positive spike which is the same as that in the single species plasmas case. It moves at a constant velocity due to the flattop laser profile and thus the velocities of the both reflected ions are almost the same.

Figure 2 shows the case of the LP laser pulse, in which the other parameters are the same as those in Fig. 1. Compared with the case of the $\mathrm{CP}$ laser pulse, the heating effect is much more obvious. The electrostatic wave is excited and it is not intense enough to accelerate protons, or even the heavy ions. At a later time $t=180 T$ as shown in Fig. 2(b), it is found that three parts of protons are accelerated. Two parts are at the front of the target, including the forward and backward protons. The other is at the rear, which is the well-known TNSA mechanism. The interaction result is almost the same as that in the pure proton plasmas case by a LP laser pulse, but obviously worse than that in Fig. 1. On the other hand, heavy ions are accelerated much more inefficiently. Therefore, we con- clude that the CP laser pulse is very important for the acceleration of heavy ions in the present mechanism.

\section{B. Influence of charge-mass ratio and heavy ions proportion on its acceleration}

According to Eqs. (1) and (2), the same velocity of the ions with different charge-mass ratios also can be estimated as

$$
\frac{v_{i}}{c} \approx 2 \sqrt{\frac{n_{c}}{n_{e 1} A_{1} / Z_{1}+n_{e 2} A_{2} / Z_{2}}} \sqrt{\frac{m_{e}}{m_{p}}} a,
$$

which is reduced to that given in Ref. [25] for a single species target. We can rewrite Eq. (4) in terms of the ratios $\alpha=n_{e 1} / n_{e 2}$ and $\beta=\left(Z_{1} / A_{1}\right) /\left(Z_{2} / A_{2}\right)$ as

$$
v_{i} / c \approx 2 \sqrt{1+\frac{\alpha(\beta-1)}{\alpha+\beta}} \sqrt{\frac{Z_{2}}{A_{2}} \frac{m_{e}}{m_{p}} \frac{n_{c}}{n_{e}}} a,
$$

so that the effect of these ratios on the acceleration efficiency is more clear. For comparison, various combinations of $\alpha$ and $\beta$ values are used for simulation. The CP laser amplitude rises from zero to $a=2$ in $6 T$, and then remains constant. The simulation box is $101 \lambda$ in the $x$ direction, and the cold target initially occupies the region between $x=80.5 \lambda$ and $x=82.5 \lambda$. The initial total electron density is $n_{e}=5$, and the densities of the two ion species are given by $n_{e 1}=n_{e 2}=0.5 n_{e}$. The results are shown in Fig. 3. There is excellent agreement between the simulation and calculation from Eq. (5), and it is found that this shock acceleration mechanism exists for a wide parameter range. Figure 3(a) shows that, in order to raise the energy of the heavy ions efficiently, its proportion must be much lower than that of the light ions. On the other hand, since the ion velocity increases quickly at first and then saturates slowly to the maximum with the density ratio, the proportion of heavy ions need not be reduced greatly in order to obtain a significant energy increase. This point is important since the number of energetic heavy ions per bunch should not be too small. For example, for $n_{e 1} / n_{e 2}=$ 9 , or a carbon ion density of $10^{20} \mathrm{~cm}^{-3}$, the velocity is
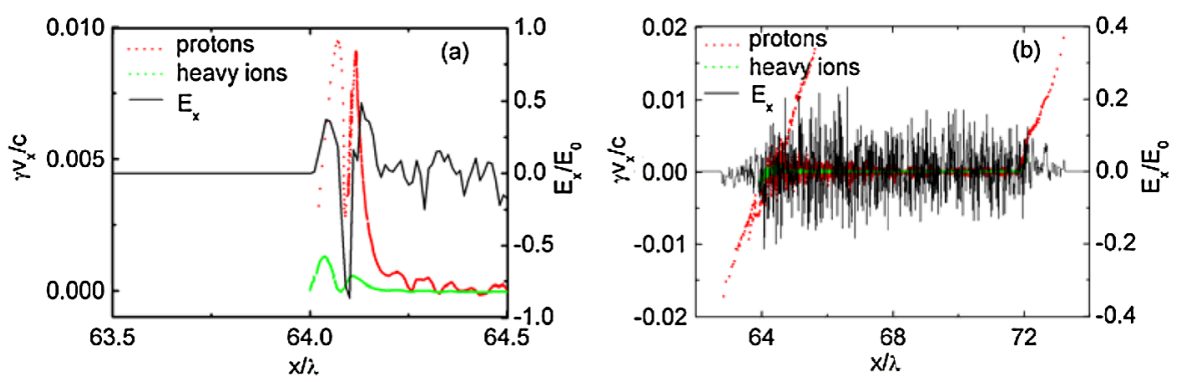

FIG. 2. (Color) Phase space of the light (red dots) and heavy ions (green dots) and the electric field distribution (black solid line) for the proton-dominated plasmas in the case of the density ratio $n_{i 1} / n_{i 2}=4$ at (a) $t=80 T$ and (b) $t=180 T$, respectively, for LP laser pulse. 

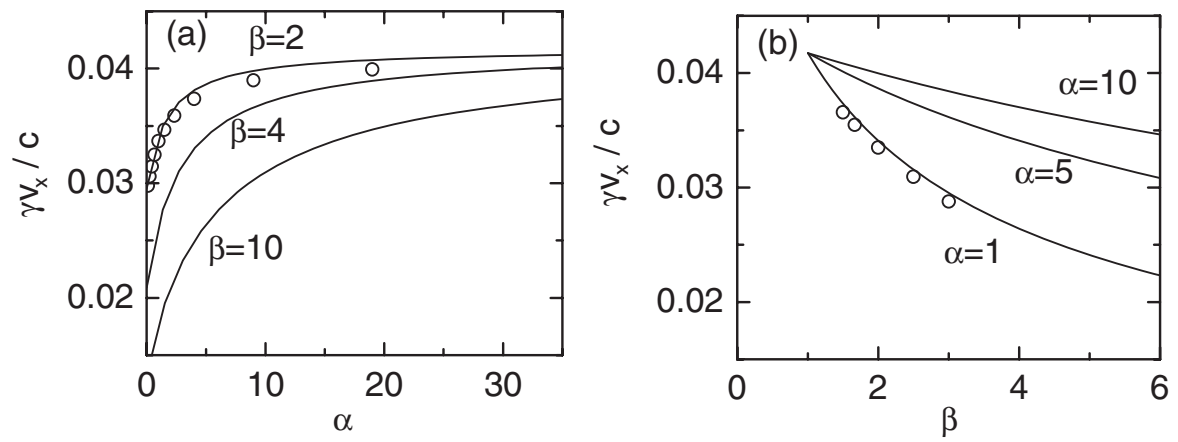

FIG. 3. Reflected heavy-ion momentum versus (a) $\alpha$ and (b) $\beta$ from simulations (circles) and Eq. (5) (solid line) at $t=150 T$.

$0.039 c$, improved by $3.4 .5 \%$ with respect to the pure carbon plasmas case, or $81 \%$ of the total kinetic energy increase. This value is close to the maximum of $41.5 \%$ predicted by Eq. (5), which is based on the steady-state conservation arguments.

To illustrate the influence of the heavy-ion proportion on its acceleration, another case with $\beta=10$ is presented. According to Eq. (3), the velocity profile of the two species of accelerated ions $v_{i 1}=v_{i 2}=v_{i}$ as a function of the proton density is shown in Fig. 4, which displays the good agreement with the simulations when $a=2, n_{e}=$ 10. That is to say, the acceleration effect of the heavy ions is better with a larger density ratio of the light and heavy ions. The maximum and minimum velocities correspond to these in the cases of pure proton and pure-heavy-ion plasmas. From the simulations, it is also found that the time for both species of ions begins to move as the same velocity grows longer with the rise of proton proportion, which means that the first stage of proton acceleration becomes longer. On the other hand, the velocity curves of heavy ions appear due to its lesser number.

It should be stressed that the results in this paper are quite different from that of Robinson et al [14] and Klimo

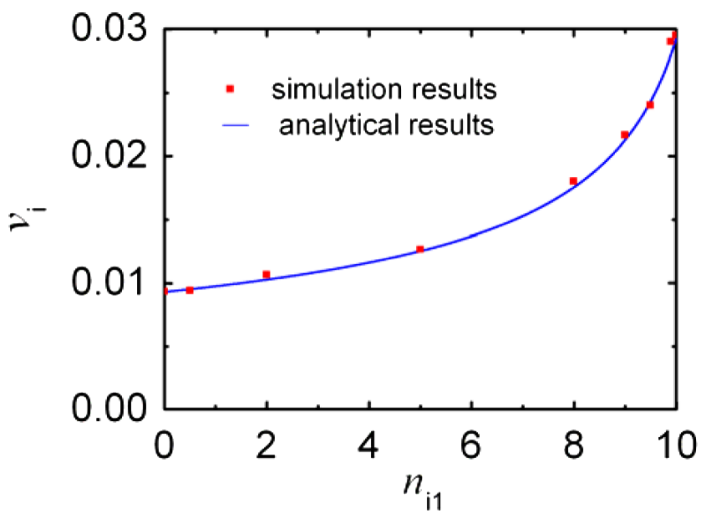

FIG. 4. (Color) Profile of the ion velocity $v_{1}$ as the function of proton density $n_{i 1}$. The red square denotes the simulation results and the blue line denotes the analytical results. et al [15] who have described a similar mechanism. The former demonstrated that the acceleration of heavy ions was not significantly affected with the final energy lower slightly than those in pure-heavy-ions case and the protons were badly affected with final energy lower greatly than those in pure protons case. The latter described simply the acceleration of a mixed ultrathin target and showed that the ballistic evolution regime of the target might be efficient for accelerating the heavy ions. They have concluded that the acceleration is easier in contrast to the TNSA mechanism. Here, in this paper we present a much more detailed and clear explanation by using quite different parameters.

\section{Improvement of the target and laser pulse}

In the above consideration, the laser pulse is flattop and thus the accelerated ions are monoenergetic. However, in most practical cases the laser is roughly Gaussian and plasma density is not a constant in space, so that according to our model used in Eq. (4), the accelerated ions may not be monoenergetic. Based on the above consideration, a simple but effective method is proposed to overcome this problem. We employ a sandwich target with a thin mixed layer between two light ion layers, as seen in Fig. 5(a). With this target structure, the rising edge of the laser pulse interacts with the front light ion layer and then the following most intense part with much shorter duration irradiates the thin mixed layer, leaving the rest to the back light ion layer. As the inside mixed layer is very thin, its interaction time with partial laser pulse is short enough that laser intensity keeps almost constant, which will lead to a more monoenergetic heavy-ion bunch.

Here the position and thickness of the thin mixed layer have great influence on the quality of heavy-ion bunches. As for Gaussian pulse laser with the intensity peaks in the beam center, it is better to place the mixed layer in the appropriate position of the sandwich target in order to make it interact with only the central part of the laser pulse, and thus ions will gain peak energy. Moreover, one can control the interaction time of the thin mixed layer and laser pulse by changing the mixed layer thickness according to the FWHM of Gaussian pulse to gain monoenergetic 
(a)

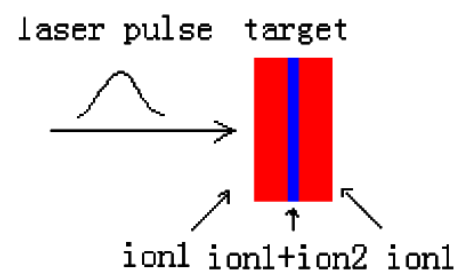

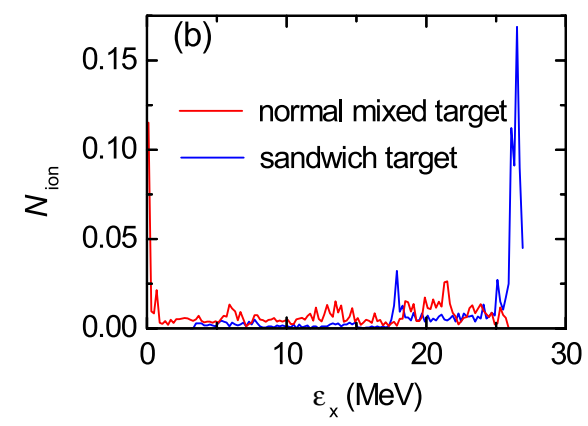

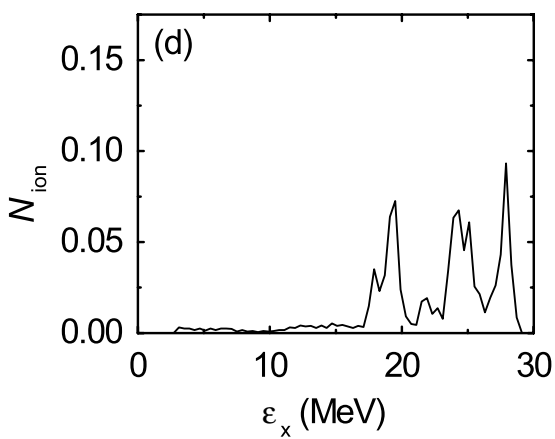

FIG. 5. (Color) (a) Sandwich target scheme and (b) the heavy-ion energy spectrums from the normal mixed target (red solid) and sandwich target (blue solid) at $t=240 T$ for a Gaussian laser pulse with peak amplitude $a=4$ and FWHM of 22T. The inside central mixed layer (hydrogen and carbon) is $0.2 \lambda$ thick with $\alpha=1$ and the outer two light ion layers (hydrogen) are both $0.9 \lambda$ thick. (c) Energy spectrum of sandwich target with the inside central mixed layer are $0.1 \lambda$ and (d) $0.4 \lambda$, respectively.

heavy-ion bunches. In a word, main parameters of heavy ions' energy spectrum can be regulated. A single thin mixed foil can not work like this because it will collapse due to the prepulse of the laser and the rising edge of the pulse. Moreover, it is more convenient to produce this sandwich target than a single thin mixed foil. The two outside layers are made up of light ions to separate the accelerated heavy ions from the other ions due to their different charge-mass ratio.

For 1D simulations, the cold sandwich target electron density is $5 n_{c}$ and the target is from $x=80.5 \lambda$ to $x=$ $82.5 \lambda$, while the simulation box is $101 \lambda$ long. The central thin layer is a mixture of hydrogen and carbon $(\beta=2)$ with their electron density ratio $\alpha=1$. Its thickness is $0.2 \lambda$ and both the front and back hydrogen layers are $0.9 \lambda$. The Gaussian laser pulse is of peak amplitude $a=4$ and FWHM 22T. A comparison of the heavy-ion kinetic energy spectrum from the sandwich target with that from the normal mixed target is shown in Fig. 5(b). All figures are taken at $t=240 T$ when the interaction between the laser and the target is just over. An obvious improvement with the sandwich target is that almost all carbon ions are around peak energy $26 \mathrm{MeV}$ while in the other case they are nearly averagely distributed. Parameters we chose here are to guarantee that the mixed layer interacts only with the center part of Gaussian pulse within a short time, and therefore heavy ions are well monoenergetic with peak energy. The thickness of mixed layer is a critical factor for the acceleration result. If the mixed layer is too thin, the heavy ions will not be fully accelerated; but if the mixed is too thick, the monoenergetic quality of the heavy ions is not perfect though the obtained maximum energy is higher, as seen in Figs. 5(c) and 5(d). Therefore, the thickness of the mixed should be controlled well to obtain the possible high monoenergetic heavy-ion bunch.

\section{3D simulations}

However, one may doubt the efficiency of the monoenergetic heavy-ion bunch generation in practice because so far only the 1D results are presented. In fact, 2D PIC simulations in Ref. [24] confirm that 2D effects do not have a qualitative influence on ion bunch formation, but they do affect energy and beam divergence of ion bunches. Following the sandwich target way, the transverse dimension of the inside thin mixed layer is reduced to weaken these negative effects. Thus, the target is embedded with a micromixed layer. The following is the 3D PIC simulations by VORPAL. Here " 1 " and " 2 " still note hydrogen and carbon. The simulation box is $25 \lambda \times 120 \lambda \times 120 \lambda$. The microstructured target occupies a region of $15 \lambda \sim 17 \lambda$ $(2 \mu \mathrm{m})$ in $x$ (longitudinal) and $-54 \lambda \sim 54 \lambda(108 \mu \mathrm{m})$ in both $y$ and $z$ (transverse) with total electron density of $5 n_{c}$. The inside mixed layer with $\alpha=1$ occupies a region of $15.4 \lambda \sim 15.435 \lambda(35 \mathrm{~nm})$ in the longitudinal direction 

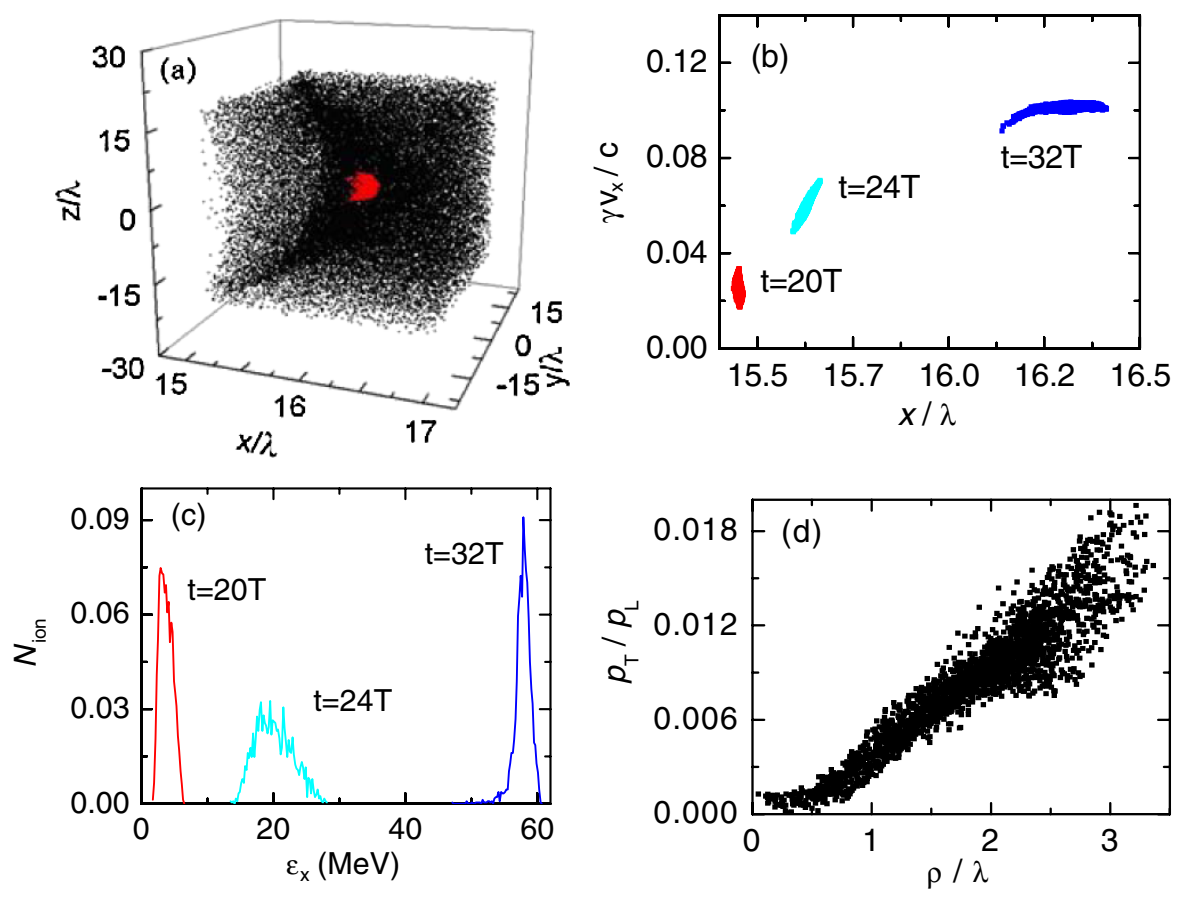

FIG. 6. (Color) (a) Space distribution of hydrogen (black dots) and carbon ions (red dots) at $t=30 T$, (b) phase space of heavy ions at different time, (c) energy spectrum of heavy ions at different time, and (d) transverse distribution of $p_{L} / p_{T}$, where $p_{L}=\gamma m_{i} v_{i x}$ and $p_{T}=\gamma m_{i}\left(v_{i y}{ }^{2}+v_{i z}{ }^{2}\right)^{1 / 2}$ are heavy ions' longitudinal and transverse momentum, respectively, and $\rho=\left(y^{2}+z^{2}\right)^{1 / 2}$ is the distance from the target center.

and $-2.4 \lambda \sim 2.4 \lambda(4.8 \mu \mathrm{m})$ in the transverse direction. The rest of the target is made up of light ions. The Gaussian laser pulse with beam waist radius $\omega_{0}=17 \lambda$, peak amplitude $a=6$, and duration of $40 T$ propagates from the left direction. Figure 6(a) shows the proton (black) and carbon ion (red) space distribution at $t=30 T$ where protons in the outer part of the target are eliminated to display the inside structure. A gauss-like shape of proton front is formed due to the Gaussian intensity profile in the transverse direction of the laser front, and all carbon ions have been reflected, leading to a compact beam with a very small dimension. Figures 6(b) and 6(c) are the phase space and longitudinal kinetic energy spectrum at different times, and we clearly see the following process: the mixed layer is initially irradiated by the laser front; carbon ions are accelerated by the electrostatic field; carbon ions are all trapped and reflected and finally propagate stably, gradually from $t=$ $20 T$ to $t=32 T$. A quasimonoenergetic carbon ion bunch with about 5\% energy divergence and the longitudinal kinetic energy of $E_{x} \sim 58 \mathrm{MeV}$ is obtained. The total carbon ion number of the bunch is about $3.36 \times 10^{8}$ (that is a total charge of $3.23 \times 10^{-10} \mathrm{C}$ ). Collimation of the obtained carbon ion bunch is pretty good as seen in Fig. 6(d), with maximum angle of divergence $\Delta \theta_{\max } \sim$ $6 \times 10^{-3} \pi \mathrm{rad}$ at the transverse rim of the mixed layer. Therefore, it can be concluded that the 3D effect does not qualitatively impact ion bunch generation. The transverse dimension of the micromixed layer can be changed to control the energy divergence.

\section{DISCUSSIONS}

\section{A. Effect of electron temperature}

Actually, an ice-cold target is usually not easily prepared. In reality, the heating effect always exists. To be more useful for experimental study, we have discussed the cases of a warm mixed target. When the target is warm, the electrostatic shock with partial ion reflection and the rest transmission will be formed [25,33], which is confirmed clearly by the results of Figs. 7(b)-7(d) in the cases of initial density $n_{e}=10$, density ratio $n_{i 1} / n_{i 2}=4$, laser amplitude $a=2$, but different initial electron thermal velocities. Ions still remain cold. From Fig. 7 we see that, when the electron temperature is relatively low, such as the electron thermal velocity $v_{\text {th }}=0.02$ in Fig. 7(a), the acceleration field is almost still a single positive spike except for some splits. The acceleration of heavy ions and protons is almost the same as that in the case of the cold target but with a slightly higher velocity, which agrees with the results of Ref. [25]. However, when the electron temperature grows higher, the thermal effect acts obviously compared with the light pressure, and the electrostatic wave is excited instead of the electrostatic field. By comparing the distributions of the electrostatic 

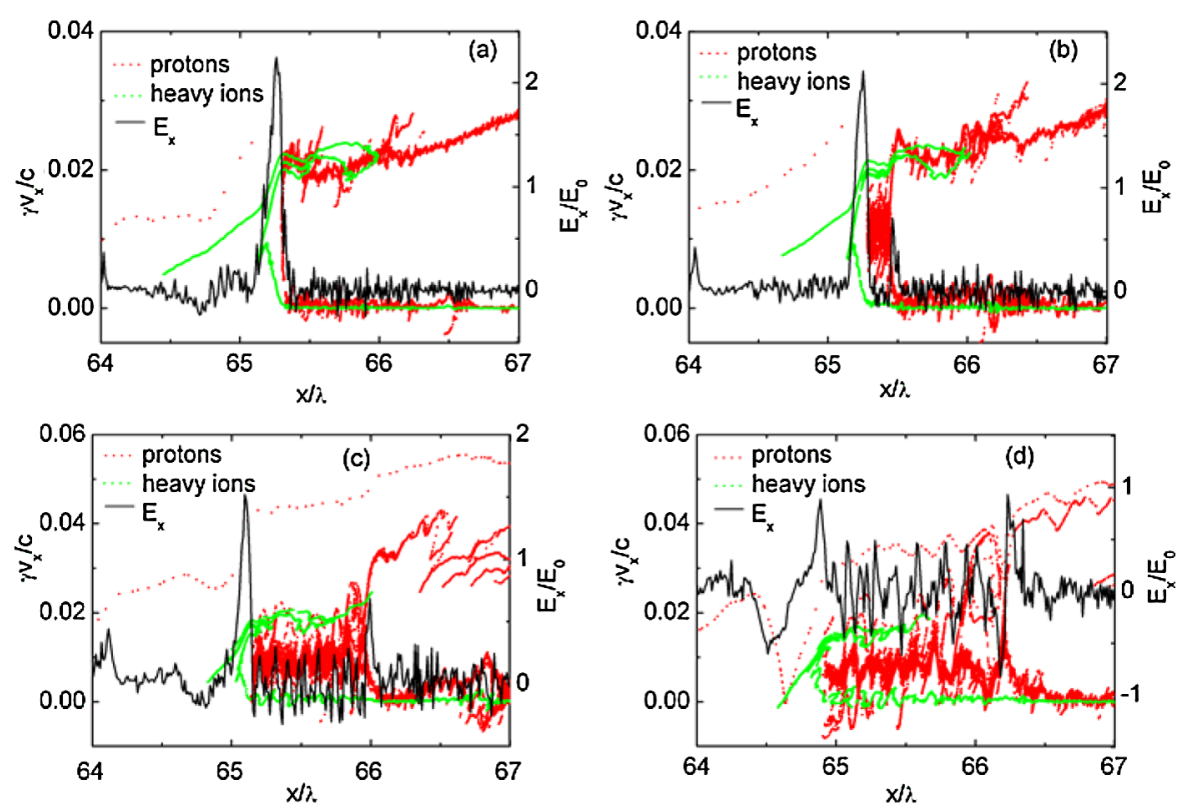

FIG. 7. (Color) Distributions of ion velocities and electrostatic field for the proton-dominated plasmas in the case of the density ratio $n_{i 1} / n_{i 2}=4$ when the initial $n_{e}=10$ at $t=180 T$ with different initial electron thermal velocities, namely, (a) $v_{\text {th }}=0.02$, (b) $v_{\text {th }}=0.04$, (c) $v_{\text {th }}=0.1$, and (d) $v_{\text {th }}=0.2$. The red and green dots represent the proton and heavy-ion velocities, respectively. The black solid line shows the electrostatic field.

field and the ions velocities, we note that protons and heavy ions are accelerated by different acceleration fields, thus resulting in different velocities. Two positive spikes are obvious in Figs. 7(b) and 7(c). As a typical electrostatic shock, more protons have transmitted the shock front with the rise of the initial electron temperature and form a soliton wavelike structure $[4,6]$. For protons, although the energy of the reflected part is much higher with the rise of the initial electron temperature, the number is fewer. For example, when the initial temperature is high enough, e.g. $v_{\text {th }}=0.2$ in Fig. 7(d), almost all protons have been transmitted and have a lower energy. However, for heavy ions, they are almost all reflected in the first acceleration field. With a low but finite initial electron temperature, the reflected velocity is slightly higher than that in the cold target case. As shown in Figs. 7(a) and 7(b), the velocity increases to 0.02. But, with the rise of the temperature, the separation field evolves into an electrostatic wave and the maximum value of the positive electrostatic field decreases, thus resulting in a smaller velocity. But it is still much more efficient than that in the pure-heavy-ions case. According to the above results, it is concluded that the low initial temperature is appropriate for the acceleration of heavy ions. Therefore, in experimental study, it is necessary to control the plasma temperature.

\section{B. Effects of target thickness and laser intensity}

As we have described, there are two stages for a mixed target, one is for light ions first and the other is subsequently for heavy ions. The acceleration process of heavy ions is almost the same as that of protons, i.e., the velocity reaches its peak and then remains constant afterward. However, if the target is thin enough, for example, the target thickness $l=0.1 \lambda$ in Fig. 8, before the velocity of heavy ions reaches its maximum, all protons immobile initially have been accelerated and subsequently are accelerated again, just as described in Ref. [25]. Heavy ions separate integrally from the protons and electrons soon. The electrostatic field has a nearly rectangular profile and there is no descending edge for heavy ions, and therefore all heavy ions are always dispersedly accelerated and

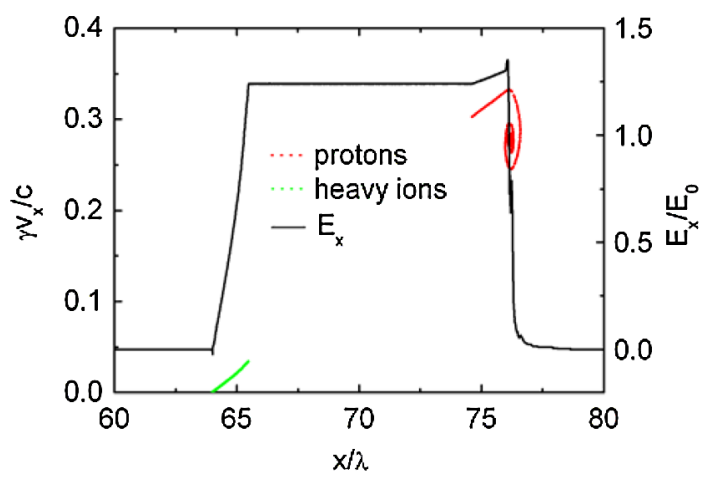

FIG. 8. (Color) Distributions of ion velocities and electrostatic field for the proton-dominated plasmas in the case of the density ratio $n_{i 1} / n_{2}=4$ when $a=2, n_{e}=10$ with the target thickness $l=0.1$ at $t=150 T$. The red and green dots represent the proton and heavy-ion velocities, respectively. The black solid line shows the electrostatic field. 


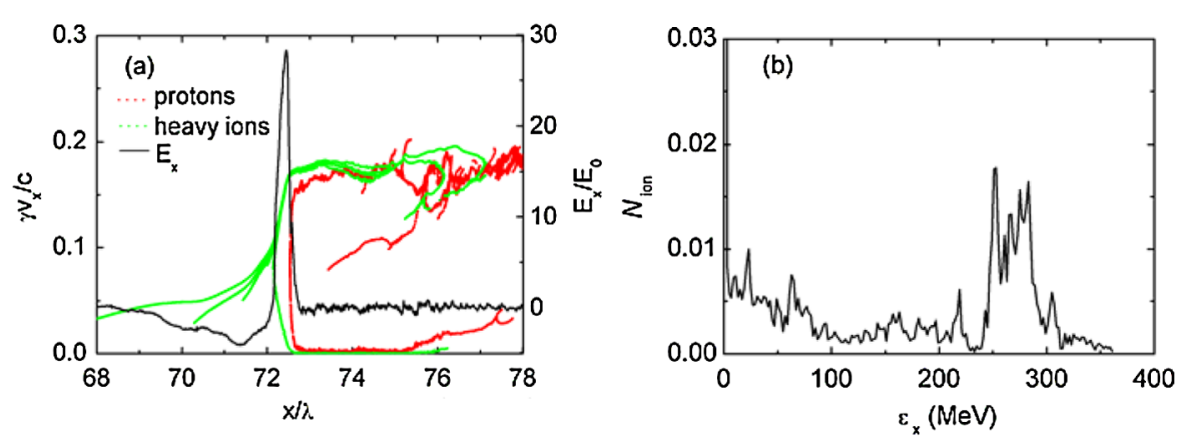

FIG. 9. (Color) Distributions of ion velocities and electrostatic field (a), and energy spectrum (b) for the proton-dominated plasmas in the case of the density ratio $n_{i 1} / n_{2}=4$ when the initial density $n_{e}=10$ and the laser amplitude $a=20$ at $t=160 T$. The red and green dots represent the proton and heavy-ion velocities, respectively. The black solid line shows the electrostatic field.

cannot be compressed like protons. Protons are accelerated to a velocity of 0.25 at $t=150 T$ just as described in Ref. [25] like a cocoon. Here, it should be noted that the long proton tail becomes shorter due to the rectangle field. So, in the case of the ultrathin target, the acceleration of heavy ions is badly affected, but accelerating protons is much more effective. We also should note that these results are quite different from that of Ref. [27] which shows that both ion species are accelerated to a nearly same velocity with a long tail in the case of ultrathin foil and ultraintense laser pulse. Here, the target is relatively much thinner.

To see the influence of the laser intensity, we have studied the case of the laser amplitude $a=20$, as shown in Fig. 9, in which the other parameters are the same as those in Fig. 1. The two species of ions are reflected to the same velocity 0.18 , ten times of that in the case of the laser amplitude $a=2$, which means that the reflected velocity is proportional to the laser amplitude, as shown in Fig. 9(a). The simulation result agrees well with Eq. (4). According to the above description, one can conclude that the more intense the laser, the better the acceleration effect of ions. However, some instability appears and the heavy-ion energy distribution becomes wider. This scenario is shown in Fig. 9(b). Then the question of how far one can go with increasing the laser intensity while preserving the ion beam quality thus remains open. In the range of normal intensity, the scaling of ion momentum with the laser intensity is applicable.

\section{SUMMARY}

In summary, when the cold plasmas consisting of two species of ions are irradiated by a CP laser pulse, there are two acceleration stages. One is for light ions and the other for heavy ions. At an early time, the light ions are accelerated efficiently by the electrostatic field due to the charge separation between electrons and light ions while the heavy ions do not have enough time to respond. At a later time, when the whole interaction becomes stable, the heavy ions and light ions are together accelerated by the quasi- stable electrostatic field and their velocities tend to be equal. The heavier ion acceleration is more efficient in the mixed plasmas than in the pure-heavy-ion plasma. Moreover, the acceleration effect is better when their proportion is lower, which agrees well with the analytical results and suggests that much more laser energy has been transferred to heavy ions. It is a new efficient acceleration approach of heavy ions though the acceleration of protons using this scheme is badly affected.

When the laser pulse is not uniform in space and time, a sandwich microstructured target with a micromixed layer in it are proposed. Using 3D PIC simulations, a quasimonoenergetic heavy-ion bunch is obtained. This method is so effective and practical that the energy of heavy ions can easily be raised by nearly $100 \%$ (or higher for heavier ions) under the same laser conditions. By using the microstructured target, the quality of the heavy-ion beam, including the energy and space divergence, is improved significantly. As an estimate, the LULI laser (wavelength $\sim 1 \mu \mathrm{m}$, intensity $\sim 10^{19} \mathrm{~W} \mathrm{~cm}^{-2}$, focal aperture $\sim 5-10 \mu \mathrm{m}$, and duration about $300 \mathrm{fs}$ ) should be able to generate a carbon beam with a peak energy of $12 \mathrm{MeV}$ and a total carbon ion number of $\sim 10^{8}$ while the energy divergence is at $5 \%$. Moreover, the fraction of carbon ions in the target can be reduced so that the maximum acceleration energy of $18 \mathrm{MeV}$ can be obtained. The discussion about the influences of the laser intensity and plasmas background temperature suggests that high laser intensity and low temperature in a certain range are appropriate for the acceleration of heavy ions. For the acceleration of protons, an ultrathin mixed target is much better.

It should be noted that the laser and plasma parameters used in the simulation are presently easily achievable in the laboratory, so that these results should be useful for experimental research on the heavy-ion beam generation. The velocities of the accelerated ions in the current simulations are relatively low due to the low laser intensity. Relativistic ions are expected when the laser intensity is further increased and the percentage of the heavy ions is optimized. 


\section{ACKNOWLEDGMENTS}

This work was supported by the 973 Program (Project No. 2006CB806004), the National Natural Science Foundation of China (Projects No. 10675155 and No. 10834008), and Scientific Research Foundation for Awarder of Excellent Doctor Thesis and President Award of Chinese Academy of Sciences (Project No. 0801051$\mathrm{X} 00)$.

[1] M. Roth et al., Phys. Rev. Lett. 86, 436 (2001).

[2] L. O. Silva, M. Marti, J. R. Davies, R. A. Fonseca, C. Ren, F. S. Tsung, and W. B. Mori, Phys. Rev. Lett. 92, 015002 (2004).

[3] M. S. Wei et al., Phys. Rev. Lett. 93, 155003 (2004).

[4] J. Denavit, Phys. Rev. Lett. 69, 3052 (1992).

[5] S. C. Wilks, W. L. Kruer, M. Tabak, and A. B. Langdon, Phys. Rev. Lett. 69, 1383 (1992).

[6] M. Chen, Z. Sheng, Q. Dong, M. He, S. Weng, Y. Li, and J. Zhang, Phys. Plasmas 14, 113106 (2007).

[7] Q. L. Dong, Z. M. Sheng, M. Y. Yu, and J. Zhang, Phys. Rev. E 68, 026408 (2003).

[8] H. Schwoerer, S. Pfotenhauer1, O. Jaeckel, K.-U. Amthor, B. Liesfeld, W. Ziegler, R. Sauerbrey, K.W.D. Ledingham, and T. Esirkepov, Nature (London) 439, 445 (2006).

[9] B. M. Hegelich, B. J. Albright, J. Cobble, K. Flippo, S. Letzring, M. Paffett, H. Ruhl, J. Schreiber, R. K. Schulze, and J. C. Fernandez, Nature (London) 439, 441 (2006).

[10] S. P. Hatchett et al., Phys. Plasmas 7, 2076 (2000).

[11] P. Mora, Phys. Rev. Lett. 90, 185002 (2003).

[12] R. A. Snavely et al., Phys. Rev. Lett. 85, 2945 (2000).

[13] Xiaoyan Liang et al., Opt. Express 15, 15335 (2007).

[14] B. J. Albright, L. Yin, B. M. Hegelich, Kevin J. Bowers, T. J. T. Kwan, and J. C. Fernandez, Phys. Rev. Lett. 97,
115002 (2006).

[15] M. Hegelich et al., Phys. Rev. Lett. 89, 085002 (2002).

[16] M. S. Wei et al., Phys. Plasmas 13, 123101 (2006).

[17] P. McKenna et al., Phys. Rev. E 70, 036405 (2004).

[18] T. Zh. Esirkepov et al., Phys. Rev. Lett. 89, 175003 (2002).

[19] E. Fourkal, I. Velchev, and C.-M. Ma, Phys. Rev. E 71, 036412 (2005).

[20] B. Shen et al., Phys. Rev. E 64, 056406 (2001).

[21] T. Esirkepov et al., Phys. Rev. Lett. 92, 175003 (2004).

[22] B. Shen et al., Phys. Rev. E 70, 036403 (2004).

[23] T. V. Liseikina and A. Macchi, Appl. Phys. Lett. 91, 171502 (2007).

[24] A. Macchi, F. Cattani, T. V. Liseykina, and F. Cornolti, Phys. Rev. Lett. 94, 165003 (2005).

[25] X. Zhang, B. Shen, Y. Cang, X. Li, Z. Jin, and F. Wang, Phys. Lett. A 369, 339 (2007); X. Zhang, B. Shen, X. Li, Z. Jin, and F. Wang, Phys. Plasmas 14, 073101 (2007); X. Zhang, B. Shen, M. Y. Yu, X. Li, Z. Jin, F. Wang, and M. Wen, Phys. Plasmas 14, 113108 (2007); X. Zhang, B. Shen, X. Li, Z. Jin, F. Wang, and M. Wen, Phys. Plasmas 14, 123108 (2007).

[26] A. P. L. Robinson, M. Zepf, S. Kar, R. G. Evans, and C. Bellei, New J. Phys. 10, 013021 (2008).

[27] O. Klimo, J. Psikal, J. Limpouch, and V. T. Tikhonchuk, Phys. Rev. ST Accel. Beams 11, 031301 (2008).

[28] O. Shorokhov and A. Pukhov, Laser Part. Beams 22, 175 (2004).

[29] L. Ji, B. Shen, X. Zhang, F. Wang, Z. Jin, X. Li, M. Wen, and J. R. Cary, Phys. Rev. Lett. 101, 164802 (2008).

[30] W. Yu, H. Xu, F. He, M. Y. Yu, S. Ishiguro, J. Zhang, and A. Y. Wong, Phys. Rev. E 72, 046401 (2005).

[31] C. Nieter and J. R. Cary, J. Comput. Phys. 196, 448 (2004).

[32] W. Yu, M. Y. Yu, J. Zhang, and Z. Xu, Phys. Rev. E 58, 6553 (1998).

[33] D. A. Tidman and N.A. Krall, Shock Waves in Collisionless Plasmas (Wiley-Interscience, New York, 1971), pp. 99-112. 